\title{
Unchanged muscle fiber conduction velocity relates to mild acidosis during exhaustive bicycling
}

\author{
J. P. J. Schmitz • J. P. van Dijk · P. A. J. Hilbers • \\ K. Nicolay $\cdot$ J. A. L. Jeneson $\cdot$ D. F. Stegeman
}

Received: 23 May 2011/Accepted: 5 August 2011/Published online: 23 August 2011

(C) The Author(s) 2011. This article is published with open access at Springerlink.com

\begin{abstract}
Muscle fiber conduction velocity (MFCV) has often been shown to decrease during standardized fatiguing isometric contractions. However, several studies have indicated that the MFCV may remain constant during fatiguing dynamic exercise. It was investigated if these observations can be related to the absence of a large decrease in $\mathrm{pH}$ and if MFCV can be considered as a good indicator of acidosis, also during dynamic bicycle exercise. High-density surface electromyography (HDsEMG) was combined with read-outs of muscle energetics recorded by in vivo ${ }^{31} \mathrm{P}$ magnetic resonance spectroscopy (MRS).
\end{abstract}

Communicated by Håkan Westerblad.

J. P. J. Schmitz $(\bowtie)$ · K. Nicolay · J. A. L. Jeneson

Biomedical NMR, Department of Biomedical Engineering,

Eindhoven University of Technology, P.O. box 513,

5600MB Eindhoven, The Netherlands

e-mail: J.P.J.Schmitz@ tue.nl

J. P. J. Schmitz · P. A. J. Hilbers

Biomodeling and Bioinformatics, Department of Biomedical

Engineering, Eindhoven University of Technology,

P.O. box 513, 5600MB Eindhoven, The Netherlands

J. P. van Dijk - D. F. Stegeman

Department of Neurology, Donders Institute for Brain, Cognition and Behaviour, Centre for Neuroscience,

Radboud University Nijmegen Medical Centre,

Nijmegen, The Netherlands

J. A. L. Jeneson

Child Development and Exercise Center,

Wilhelmina Kinderziekenhuis/University Medical Center

Utrecht, Utrecht, The Netherlands

D. F. Stegeman

Research Institute MOVE, Faculty of Human Movement

Sciences, VU University, Amsterdam, The Netherlands
Measurements were performed during serial exhausting bouts of bicycle exercise at three different workloads. The HDsEMG recordings revealed a small and incoherent variation of MFCV during all high-intensity exercise bouts. ${ }^{31} \mathrm{P}$ MRS spectra revealed a moderate decrease in $\mathrm{pH}$ at the end of exercise ( $\sim 0.3$ units down to 6.8$)$ and a rapid ancillary drop to $\mathrm{pH} 6.5$ during recovery $30 \mathrm{~s}$ post-exercise. This additional degree of acidification caused a significant decrease in MFCV during cycling immediately after the rest period. From the data a significant correlation between MFCV and $\left[\mathrm{H}^{+}\right] \quad\left(\left[\mathrm{H}^{+}\right]=10^{-\mathrm{pH}}\right)$ was calculated ( $p<0.001$, Pearson's $R=-0.87$ ). Our results confirmed the previous observations of MFCV remaining constant during fatiguing dynamic exercise. A constant MFCV is in line with a low degree of acidification, considering the presence of a correlation between $\mathrm{pH}$ and MFCV after further increasing acidification.

Keywords Skeletal muscle $\cdot{ }^{31} \mathrm{P}$ magnetic resonance spectroscopy $\cdot$ High-density surface EMG $\cdot$ Dynamic exercise $\cdot$ MFCV

\section{Introduction}

Surface electromyography (sEMG) is widely used to study the electrical activity of motor units within active skeletal muscle (Farina et al. 2004a). Mean muscle fiber conduction velocity (MFCV) can be estimated non-invasively from the sEMG recordings of multiple closely spaced electrodes. MFCV is known to be influenced by multiple physiological and, or environmental factors. It varies with mean muscle fiber diameter and muscle fiber type, as well as the level of force output (Zwarts and Stegeman 2003). An increase in MFCV is often explained by recruitment of larger motor 
units or may be a result of a rise in muscle temperature. During sustained isometric exercise at high-contraction levels [40-100\% maximal voluntary contraction (MVC)], a decrease in MFCV has often been reported (e.g., ArendtNielsen and Mills 1988; Krogh-Lund 1993; Masuda et al. 1999; Zwarts et al. 1987). A combination of high-metabolic demand, fast motor unit firing rates, and diminution of the circulation with the accumulation of metabolic byproducts gives rise to a progressive change in the membrane microenvironment (Miller et al. 1995). The accumulation of extracellular potassium, especially in the T-tubulus, and the accumulation of lactate together with a lowering of $\mathrm{pH}$ seem to be the main determinants for the lowering of MFCV (Zwarts and Stegeman 2003). In and by itself, a lower MFCV is not likely to decrease the force generating capacity of the muscle (Allen et al. 2008). However, some factors involved in the development of muscle fatigue, e.g., muscle acidification (Allen et al. 2008) and potassium accumulation (Fortune and Lowery 2009) have also been proposed to affect MFCV (Zwarts and Stegeman 2003). Such a common source of both muscle fatigue and decreasing MFCV would explain why these phenomena often coincide. For example, Linssen et al. (1996) measured MFCV in a group of McArdle's patients who lack the ability to consume glycogen in the glycolytic pathway. These patients showed no change in muscle $\mathrm{pH}$ during fatiguing exercise, and, in addition no decrease in MFCV. Houtman et al. $(2001,2003)$ observed a similar association between muscle $\mathrm{pH}$ changes and MFCV in healthy muscle. These data indicated muscle $\mathrm{pH}$ to be one of the factors strongly related to MFCV dynamics. MFCV recordings may, therefore, provide relevant information about muscle acidosis during exercise.

Previous investigations of MFCV dynamics focused mainly on standardized isometric tasks. The study of EMG variables during dynamic exercise is, however, of greater practical and fundamental relevance as dynamic exercise more closely resembles tasks executed during sports and everyday activity (Macdonald et al. 2008). The advent of adhesive multichannel EMG electrode grids (Lapatki et al. 2004; Merletti et al. 2009) has recently enabled MFCV recording during this type of exercise (Macdonald et al. 2008). Yet to date, only a few studies have focused on the response of MFCV during fatiguing dynamic contractions and with partly contradictory results. During coupled concentric and eccentric exercise performed on a flywheel exercise device, MFCV decreased with fatiguing exercise (Pozzo et al. 2006), but it showed no change during fatiguing repetitive knee extensions (Masuda et al. 1999). MFCV during cycling exercise was reported either to decrease (Farina et al. 2004b; Sbriccoli et al. 2009) or remain constant (Macdonald et al. 2008). These results provide no clear picture of the response of MFCV during dynamic exercise.
A possible explanation for the lack of consistency among the results of investigations of MFCV dynamics during dynamic exercise compared to isometric exercise may be that the dynamics of factors affecting MFCV may be very different between these regimens. For example, it has been found that the magnitude of muscle acidification during intense bicycle exercise is significantly less than that typically observed during isometric contractions (Schmitz et al. 2010). We propose that the absence of a large drop in $\mathrm{pH}$ may explain the absence of a decrease in MFCV during intense cycling exercise. This hypothesis is based on the evidence suggesting a correlation between $\mathrm{pH}$ and MFCV, as reported by Houtman et al. (2001, 2003), Linssen et al. (1996) and Zwarts and Stegeman (2003), and the data indicating only mild acidosis during intense bicycle exercise (Jeneson and Bruggeman 2004; Schmitz et al. 2010). In an attempt to substantiate the interpretation of MFCV dynamics, we investigated the relation between MFCV and $\mathrm{pH}$ dynamics during intense bicycle exercise. Specifically, we tested the hypothesis that mild acidosis in skeletal muscle does not result in a significant and detectable decrease of MFCV during bicycle exercise. MFCV, intramuscular $\mathrm{pH}$ and high-energy phosphate metabolite concentrations during different bouts of exhaustive cycling exercise were measured using high-density surface EMG, and ${ }^{31} \mathrm{P}$ magnetic resonance spectroscopy (MRS). The results of the study support the proposed hypothesis especially after employing additional post-exercise experiments which confirmed a strong correlation between MFCV and $\mathrm{pH}$.

\section{Materials and methods}

\section{Subjects}

Six healthy normally active male subjects (median age 25 years; range $21-45$ years) participated in the study. The nature and risks of the experimental procedures were explained to the subjects, and all gave their written informed consent to participate in the study, which conformed to the standards set by the Declaration of Helsinki and was approved by the local Medical Ethical Committee of the Máxima Medical Centre, Veldhoven, The Netherlands. All subjects wore shorts and athletic shoes during the examination.

\section{Exercise protocol}

The subjects performed bicycle exercise in the supine position on a custom-build non-ferrous, mechanically braked bicycle ergometer. This ergometer was designed to fit the bore of the 1.5-T whole body MRI scanner used to perform the ${ }^{31} \mathrm{P}$ MRS measurements. An image of the 
Fig. 1 Image of the MRcompatible bicycle ergometer. Parts indicated in the image are: 1 wooden flywheel, 2 mechanical brake, 3 pedals and 4 nylon transmission belt

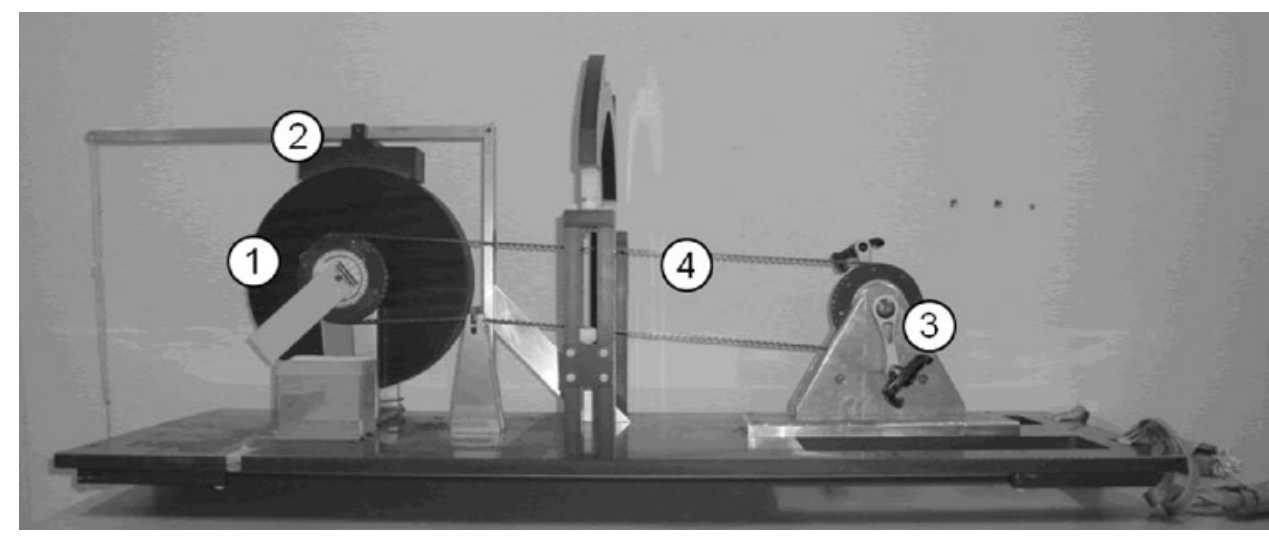

ergometer is shown in Fig. 1. Details of the ergometer are described elsewhere (Jeneson et al. 2010). In brief, the ergometer was mounted on a high-density plastic support. A central pinwheel equipped with brass bearings and pedals on $10-\mathrm{cm}$ aluminum levers was mounted on an aluminum block. A nylon transmission belt connected the pedals to a wooden flywheel. A variable braking load was applied gravimetrically by adding a number of brass weights $(500 \mathrm{~g})$ to an aluminum bucket suspended from the lever arm. The feet of the subject were fixated to the pedals with strong adhesive tape. The subjects were instructed to only apply force to the pedals during the pushing phase of the cycle. The smallest and largest knee angle was $100^{\circ}$ and $160^{\circ}$, respectively. As a result of the restriction to use only non-ferrous materials in the ergometer design, it was not possible to quantify exercise intensity in terms of absolute power output (Watts). Instead, the applied braking load in Newtons was used to quantify workload intensity.

The pedaling rate was $80 \mathrm{rpm}$, set by a metronome. To perform both the high-density sEMG and ${ }^{31} \mathrm{P}$ MRS, subjects visited the laboratory twice for a duplicate experiment. On the first day the ergometer was placed outside the bore of the MRI scanner and high-density sEMG measurements were performed. Subjects lay in a supine position on a patient bed placed behind the ergometer. It was verified that the knee angle was equal to the conditions in the bore of the MR scanner. On the second day, after a week, the ergometer was positioned in the MR scanner and the ${ }^{31} \mathrm{P}$ spectra were recorded. On both days, the protocol consisted of three experiments, each at a different braking load, 35, 50 and $65 \mathrm{~N}$, respectively (Fig. 2). The rationale behind selection of workload intensity was to define different strenuous workloads which would induce a highglycolytic flux, rapid lactate accumulation and task failure after only a few minutes of exercise. These conditions were considered optimal for rigorous testing of the proposed hypothesis. In the first two experiments ( 35 and $50 \mathrm{~N}$ ), the subjects continued exercise until task failure. Task failure was defined as the subject not being able to continue the exercise workload at $80 \mathrm{rpm}$, although being vocally encouraged, increasing the pedaling frequency to the 80-rpm cadence. In the third experiment with $65-\mathrm{N}$ load, exercise until task failure was followed by two extra bouts of $24 \mathrm{~s}$, both after $30 \mathrm{~s}$ of rest. The three experiments were performed in random order and separated by $20 \mathrm{~min}$ of rest. Post hoc ${ }^{31} \mathrm{P}$ MRS confirmed that this rest period was sufficient for a full recovery of metabolite concentrations $(\mathrm{Pi}, \mathrm{PCr}$ and $\mathrm{ATP})$ and cellular $\mathrm{pH}$ to resting levels at all three braking levels. The duration of the different exercise bouts was $240 \pm 143 \mathrm{~s}$ (range 152-280 s), $95 \pm 17 \mathrm{~s}$ (range 72-120 s) and $49 \pm 12 \mathrm{~s}$ (range 39-56 s) for the $35-, 50-$ and $65-\mathrm{N}$ workload, respectively.

\section{High-density surface EMG}

First, a high-density electrode grid with dry electrode contacts (Blok et al. 2002) was used to position and reposition iteratively on the vastus medialis of the right leg of the subjects until the electrode grid was aligned to the muscle fibers of the vastus medialis. The final position of the grid was marked on the leg of the subject with a fine liner. Next, the dry electrode grid was removed and a thin flexible multichannel electrode grid with an inter-electrode distance of $4 \mathrm{~mm}$ was attached to the skin with a $100-\mu \mathrm{m}$ thick double-sided adhesive tape, as described elsewhere (Lapatki et al. 2004). The common-mode-sense and reference electrodes were placed distally from the grid on the patella of the right knee. A "driven-right-leg" electrode (Metting van Rijn et al. 1990) was placed on the tibia of the right leg. Monopolar signals were amplified, band pass filtered $(0.16-500 \mathrm{~Hz})$ and simultaneously analog-to-digital converted using a multichannel amplifier system (Active-One, BioSemi, Amsterdam, The Netherlands) at a rate of 2,048 samples s ${ }^{-1}$ channel $^{-1}$. Signals were highpass filtered off-line at $10 \mathrm{~Hz}$ using a second order Butterworth filter. 
Fig. 2 Schematic overview of the exercise protocol at workload intensities of 35-, $50-$ and $65-\mathrm{N}$ braking force. Duration of the first exercise bout lasted until exhaustion or until the pedaling rate of $80 \mathrm{rpm}$ could no longer be met. The order of the three workloads was randomly chosen

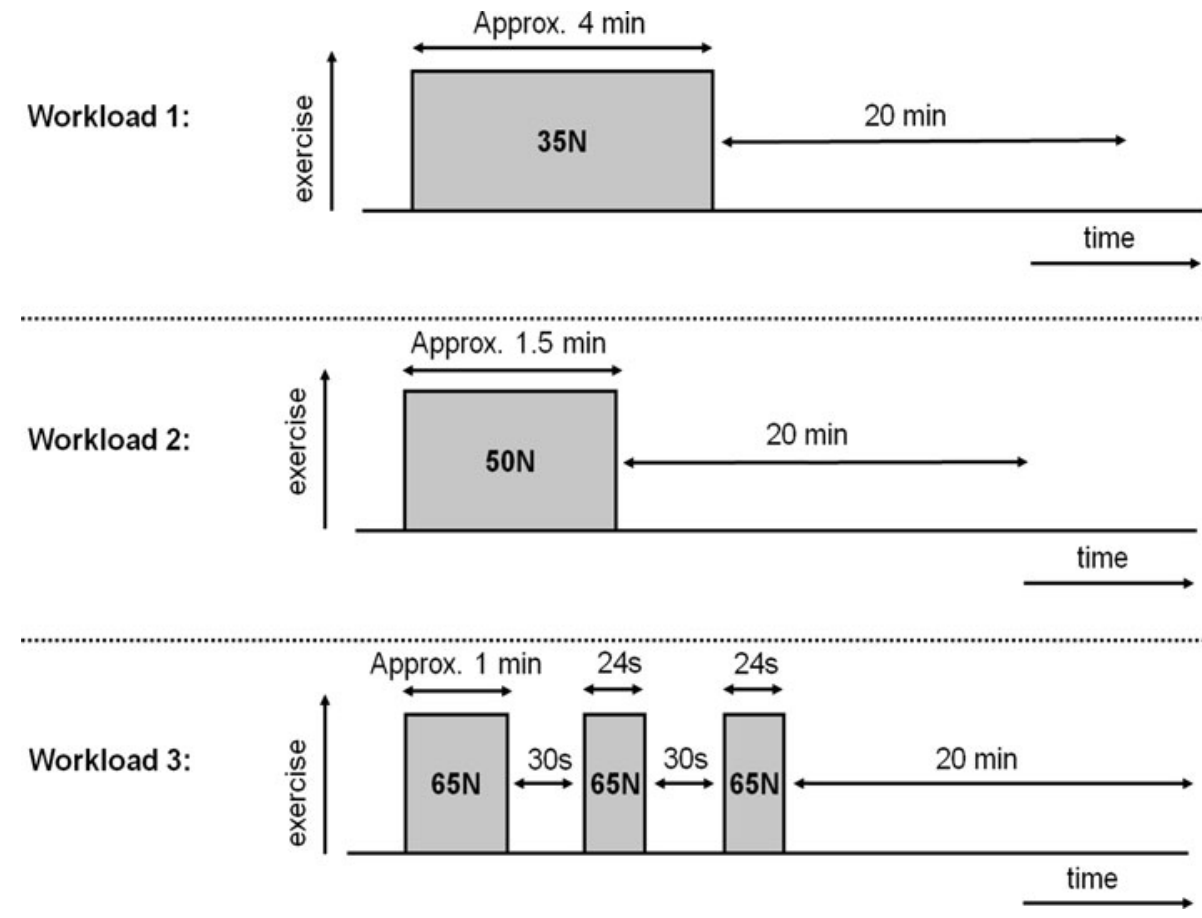

with a length corresponding to $10 \%$ of the duration of each exercise bout.

\section{${ }^{31} \mathrm{P}$ NMR spectroscopy}

The ${ }^{31} \mathrm{P}$ MRS measurements were performed on a $1.5-\mathrm{T}$ whole body scanner (Gyroscan S15/ACS, Philips Medical Systems, Best, The Netherlands). A single-turn ${ }^{31} \mathrm{P}$ surface coil (diameter $6 \mathrm{~cm}$ ) supplied by the manufacturer was positioned over the vastus medialis muscle of the right leg and fastened with Velcro straps. The medial head of the quadriceps muscle was chosen because of the most optimal magnetic field homogeneity in the center of the bore of the MR scanner. The magnet was shimmed using a custom-built rectangular ${ }^{1} \mathrm{H}$ surface coil $(20 \times 10 \mathrm{~cm})$, positioned over the quadriceps muscle as described elsewhere (Jeneson et al. 2010). All ${ }^{31} \mathrm{P}$ NMR spectra were acquired using a $90^{\circ}$ adiabatic excitation pulse. Prior to the exercise bout, two ${ }^{31} \mathrm{P}$ spectra (16 summed free induction decays (FIDs); 1,024 points; sweep width $2 \mathrm{kHz}$ ) were obtained from the resting muscle in resting position under fully relaxed (repetition time (TR) $15 \mathrm{~s}$ ) and partly-saturated (TR $3 \mathrm{~s}$ ) conditions. These spectra were used to determine saturation correction factors for each resonance. During acquisition two scans were summed. Post-acquisition, additional summation was performed by calculating a moving average of two spectra ( 4 scans).

\section{Data analysis}

Spectra were fitted in the time domain by using a nonlinear least-squares algorithm (AMARES) in the jMRUI software 
package (Vanhamme et al. 1997). Pi, PCr and ATP signals were fitted to lorentzian line shapes. The three ATP peaks were fitted as two doublets and one triplet, with equal amplitudes and line widths and prior knowledge for the J-coupling constant $(17 \mathrm{~Hz})$. Maximal line widths were constrained at $30 \mathrm{~Hz}$. Absolute concentrations of the metabolites were calculated after correction for partial saturation and assuming that the ATP concentration ([ATP]) is $8.2 \mathrm{mM}$ at rest (Taylor et al. 1986). Intracellular $\mathrm{pH}$ was calculated from the chemical shift difference between the $\mathrm{Pi}$ and $\mathrm{PCr}$ resonances $(\delta$; measured in parts per million) according to Eq. 1 (Tobin et al. 1972):

$\mathrm{pH}=6.75+\log \left(\frac{\delta-3.27}{5.63-\delta}\right)$

Statistical analysis

All statistical analyses were performed with PASW statistics 18 (SPSS Inc, Chicago, Illinois, USA). Unless otherwise stated, the statistical significance of observations was determined using a 0.05 chance threshold by performing ANOVA for repeated measures, followed by a Bonferroni's post hoc analysis.

\section{Results}

Muscle fiber conduction velocity

The mean MFCV for each workload was calculated for all individual subjects (Fig. 3). For all subjects the expected trend of increasing MFCV at higher workloads was observed. The significance of this observation was confirmed as indicated in Fig. 3.

The normalized time dynamics of MFCV were calculated as described in the "Materials and methods" section. The experimental data were pooled per workload $(n=6)$ and are shown in Fig. 4. The results shown for the $65-\mathrm{N}$ workload correspond to the first exercise bout at this intensity. From these results it was observed that the MFCV remained constant during each workload. It was calculated that for each workload, there was no significant difference between the MFCV at the onset of exercise (first data point) and at the end of exercise (last data point) ( $p \geq 0.15$, statistical test: paired $t$ test). MFCV time dynamics were also analyzed by performing ANOVA for repeated measures with Bonferroni post hoc analyses (significance threshold set at 0.05). Pair wise comparison of the means revealed no statistically significant difference between all possible combinations of two data points, except for one $(65-\mathrm{N}$ workload, 6th and 8th data points). These results do not indicate a general trend of increasing or decreasing MFCV
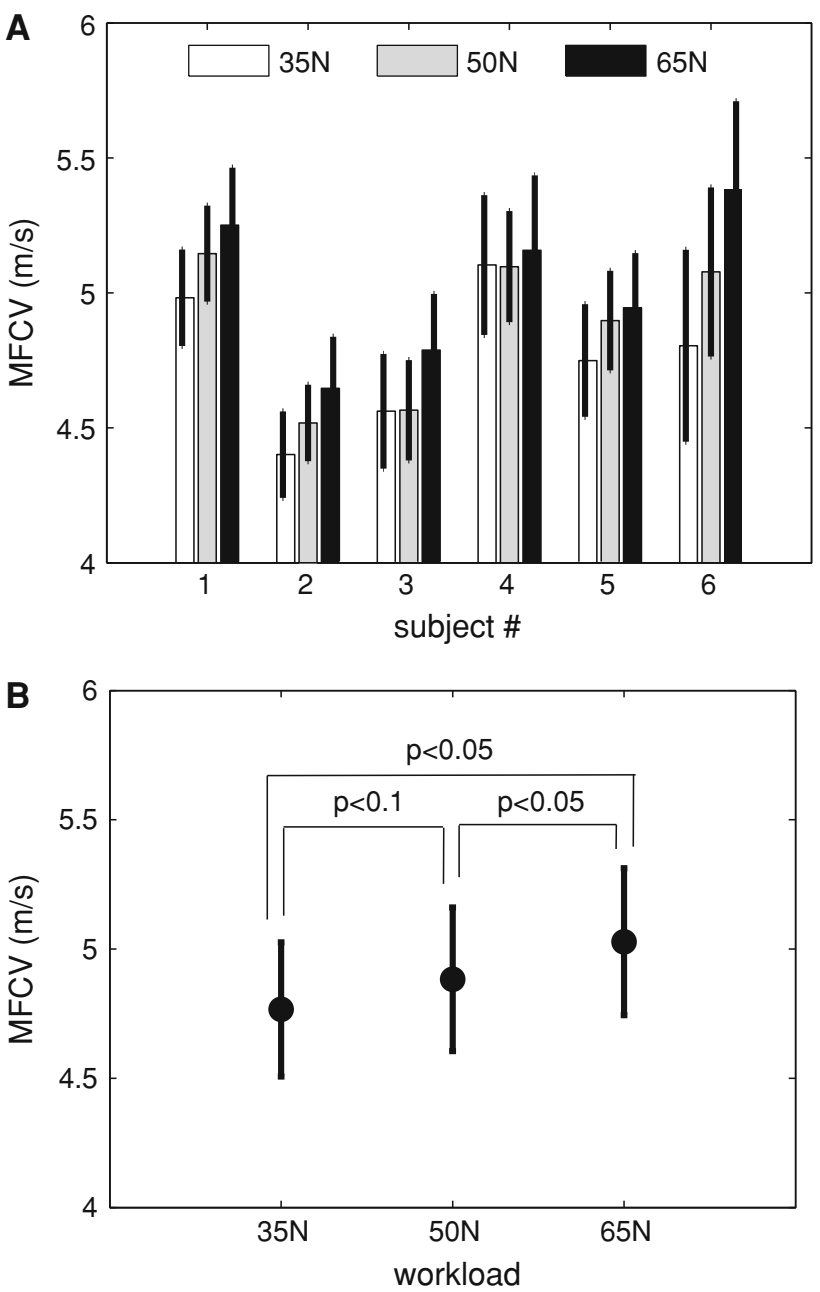

Fig. 3 Mean muscle fiber conduction velocity (MFCV) for individual subjects (a) and pooled per workload (mean $\pm \mathrm{SD}, n=6)(\mathbf{b})$

during the exercise bouts and therefore, they were considered further evidence that, except for some small and inconsistent variations, the MFCV remained constant during individual workloads. In addition, a linear regression model was fitted to the data of the individual subjects. The slope of the linear regression model corresponded to an average decrease in MFCV over all three braking loads of $0.1 \pm 3.4 \%$ (mean $\pm \mathrm{SD}$ ) for the entire duration of the workloads, which clearly is not different from zero.

\section{${ }^{31} \mathrm{P}$ NMR spectroscopy}

PCr concentration decreased from $33.4 \pm 1.5 \mathrm{mM}$ at rest to $4.3 \pm 2.01,5.9 \pm 1.4$ and $4.9 \pm 1.2 \mathrm{mM}$ at the end of exercise at braking loads of 35, 50 and $65 \mathrm{~N}$. This nearly full depletion was accompanied by a complementary increase in $[\mathrm{Pi}]$, whereas ATP concentration remained constant, data not shown. There was found no significant difference in end exercise [PCr] between different loads 

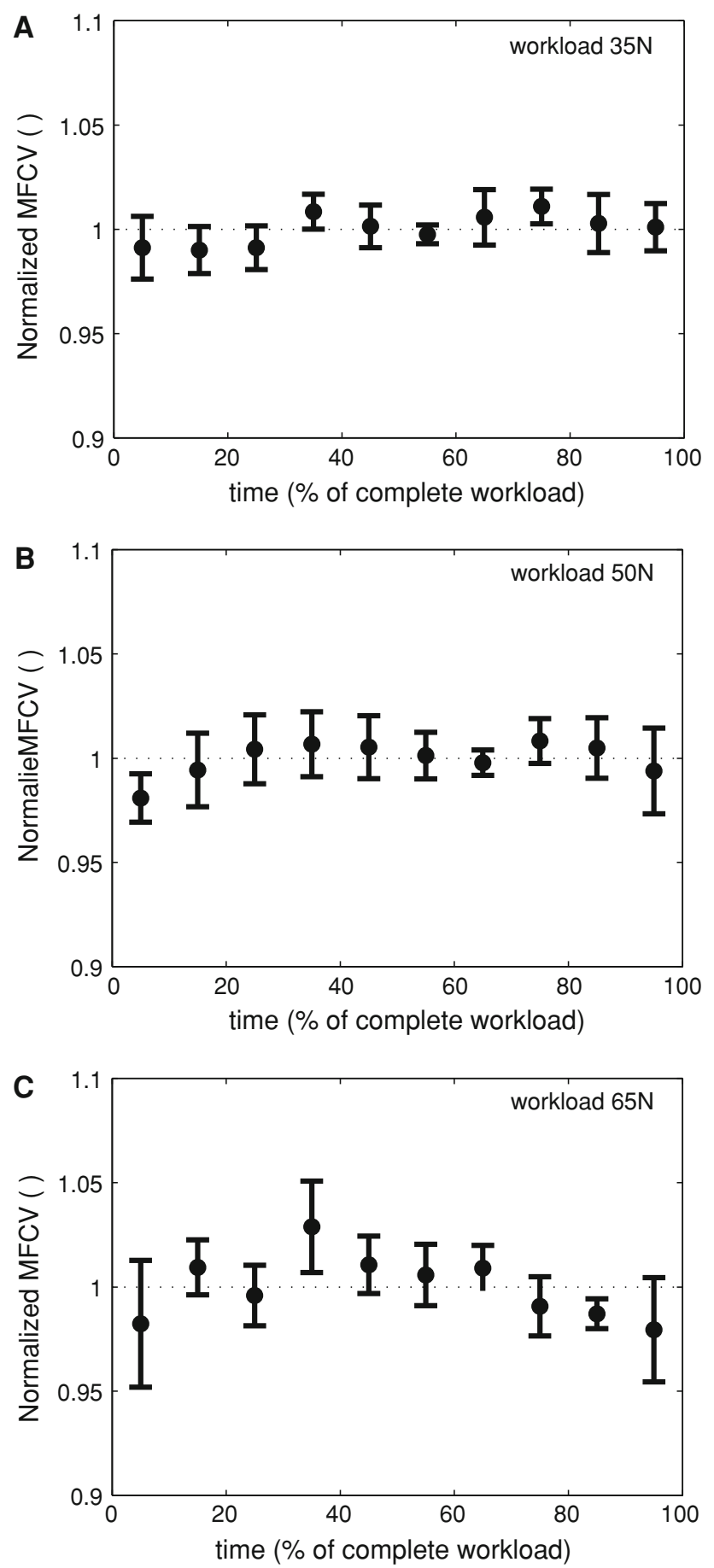

Fig. 4 Normalized muscle fiber conduction velocity (MFCV) during exercise at with a braking load of $35 \mathrm{~N}(\mathbf{a}), 50 \mathrm{~N}(\mathbf{b})$ and $65 \mathrm{~N}(\mathbf{c})$. Data points represent mean $\pm \mathrm{SD}(n=6)$. MFCV data were normalized with respect to the mean MFCV

$(p \geq 0.15)$. The $\mathrm{pH}$ decreased from $7.08 \pm 0.05$ at rest to $6.79 \pm 0.18,6.80 \pm 0.06$ and $6.85 \pm 0.07$ at the end of exercise at braking loads of 35,50 and $65 \mathrm{~N}$. This mild decrease in $\mathrm{pH}$ did not significantly differ between workloads $(p \geq 0.8)$.
MFCV and $\mathrm{pH}$ during three exercise bouts at $65 \mathrm{~N}$

As indicated, the first exercise bout at $65 \mathrm{~N}$ was followed by two periods consisting of a sequence of $30 \mathrm{~s}$ rest and $24 \mathrm{~s}$ exercise. The MFCV and $\mathrm{pH}$ dynamics during the three exercise bouts at $65 \mathrm{~N}$ protocol are shown in Fig. 5a, b. The MFCV during the first exercise bout is identical to the data shown in Fig. 4c. Obviously, during the rest period following the first and second exercise bout no MFCV could be estimated. However, at the onset of the second and third exercise bout a significant decrease in MFCV was detected compared to the MFCV at the end of the previous exercise bout ( $p<0.05$, paired $t$ test). Moreover, during the second and the third exercise bout, a similar trend of increasing MFCV during these exercise bouts was observed. For each individual subject it was tested if the slope of a linear regression model was positive and significantly different from zero (Table 1). It was found that for all subjects in both the second and the third exercise bout the slope of the linear model was significantly different from zero $(p<0.05)$ at a value of $1.62 \pm 0.25$ and $1.64 \pm 0.72 \mathrm{~m} \mathrm{~s}^{-1} \mathrm{~min}^{-1}$, respectively (mean $\pm \mathrm{SD}, n=6$ ).

The muscle $\mathrm{pH}$ decreased during the first exercise bout from $7.02 \pm 0.13$ at rest to $6.83 \pm 0.03$ at exhaustion. Pi concentration increased from $5.9 \pm 1.8 \mathrm{mM}$ at rest to $29.3 \pm 4.6 \mathrm{mM}$ at exhaustion. Muscle $\mathrm{pH}$ and $\mathrm{Pi}$ could also be measured during the rest periods (Fig. 5b, c). During the first 30 -s rest period, $\mathrm{pH}$ dropped an additional 0.2 units to $6.63 \pm 0.08$. This drop was attributed to the rapid consumption of proton buffer, i.e., Pi (Jeneson and Bruggeman 2004) (Fig. 5c), which decreased the total cellular proton buffering capacity. During the first extra exercise bout Pi concentration increased again. As a result, also the cellular proton buffering capacity was restored which caused an increase in cellular pH. (Fig. 5c). This sequence of events was repeated during the second 30-s rest period and second extra exercise bout. During the first exercise bout $\mathrm{pH}$ dynamics decreased while cellular $\mathrm{Pi}$ concentrations increased. After the first exercise bout Pi and $\mathrm{pH}$ dynamics behaved in-phase: the decrease and increase of $\mathrm{Pi}$ and $\mathrm{pH}$ co-occurred. The $\mathrm{pH}$ decreased during the first exercise bout as a result of lactate and $\mathrm{CO}_{2}$ formation. The proton production was not fully compensated by other processes, e.g., PCr breakdown or increase in cellular proton buffering capacity, causing a net decrease in $\mathrm{pH}$ while $\mathrm{Pi}$ concentration increased. During the rest periods and short exercise bouts (24 s), accumulation of lactate and $\mathrm{CO}_{2}$ was much less (similar to the first $24 \mathrm{~s}$ of the first exercise bout). The overall $\mathrm{pH}$ dynamics were, therefore, dominated by the dynamics of the cellular proton buffering capacity which co-varies with the Pi concentration, explaining the in-phase behavior. Analysis of the relation between MFCV and $\mathrm{pH}$ during this $65-\mathrm{N}$ 

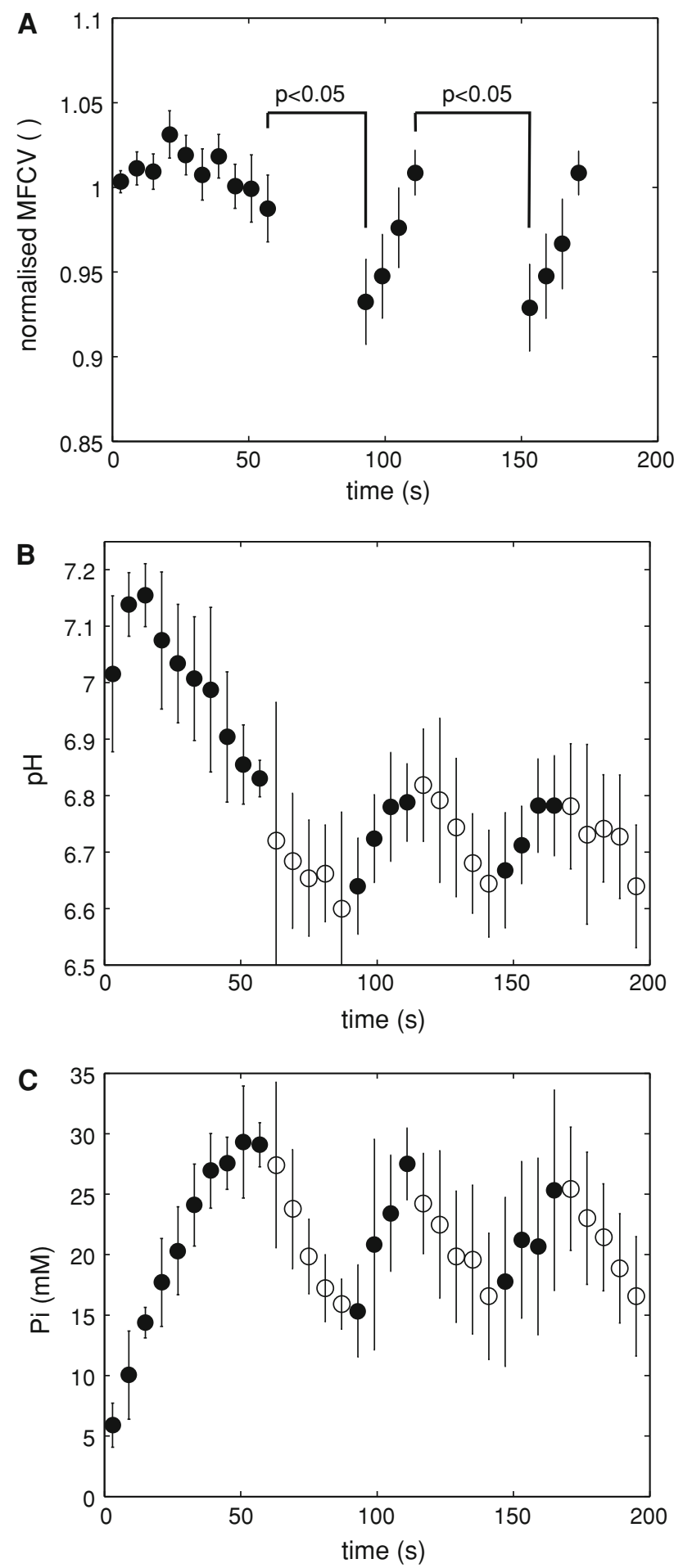

Fig. 5 Normalized muscle fiber conduction velocity (MFCV, a), $\mathrm{pH}$ (b) and inorganic phosphate concentration ([Pi], c) during the full intermittent exercise protocol ( 3 exercise bouts separated by 2 rest periods) at a workload of $65-\mathrm{N}$ braking force. Data points represent mean $\pm \mathrm{SD}(n=6)$. The data points recorded during exercise are indicated by a closed circle, the data points recorded during rest are indicated by an open circle intermittent exercise protocol (Fig. 5a, b solid black dots) revealed a significant correlation between proton concentration $\left(\left[\mathrm{H}^{+}\right]=10^{-\mathrm{pH}}\right)$, and normalized MFCV (Fig. 6) (Pearson's $R=-0.87 ; p<0.001$ ).

\section{Discussion}

A main result of this investigation was confirmation of the MFCV remaining constant during bouts of exhaustive bicycle exercise. In addition, we propose that this behavior is related to a limited drop in upper leg muscle $\mathrm{pH}$ (0.2-0.3 units). These results as well as several methodological considerations will be discussed.

Methodological considerations

The present study is unique in combining two complementary non-invasive methods. Performing the cycling exercise inside the bore of an MR scanner while recording ${ }^{31} \mathrm{P}$ spectra required a dedicated MR-compatible ergometer (Jeneson et al. 2010). Consequently, the subjects had to perform exercise in a supine position. Previously it was shown that regression lines between heart rate and $\mathrm{VO}_{2}$ and heart rate and power output did not significantly differ between supine versus upright exercise (Rodenburg et al. 1994). On this basis it was concluded that the supine exercise position was suitable for dynamic exercise testing of quadriceps muscle in a whole body MR scanner (Rodenburg et al. 1994).

The configuration of the ergometer did not allow for the quantification of exercise intensity in terms of absolute power output (Jeneson et al. 2010). For this particular investigation it was, however, critical to rule out the possibility that the absence of a decrease in MFCV was a result of a relatively moderate contribution of the vastus medialis muscle to the total power output of the legs during exhaustive bicycling. Here, the ${ }^{31} \mathrm{P}$ MRS results provided the necessary information in two ways. The first important piece of evidence for full recruitment of the vastus medialis muscle during the exercise was an almost full depletion of the PCr pool $(>80 \%)$ at the end of exercise. Depletion of the cellular ATP buffer with this high percentage requires very intense muscle contractions and has only been reported in case of very high-intensity exercise (Forbes et al. 2009; Schmitz et al. 2010; Walter et al. 1999). Secondly, the rate of $\mathrm{PCr}$ resynthesis and $\mathrm{Pi}$ consumption during post-exercise recovery provides a reliable readout of mitochondrial ATP production (Prompers et al. 2006). At the different workloads the initial rate of $\mathrm{PCr}$ recovery was $\sim 0.8 \mathrm{mM} / \mathrm{s}$, which equaled the maximal rate of mitochondrial ATP synthesis and thus respiration in human quadriceps muscle (Jeneson et al. 2009). From these results it was concluded that in these particular exercise protocols 
Table 1 Slope of increasing MFCV during second and third exercise bout at $65 \mathrm{~N}$

\begin{tabular}{llllll}
\hline Subject & \multicolumn{2}{l}{ Second exercise bout $65 \mathrm{~N}$} & & \multicolumn{2}{l}{ Third exercise bout 65 N } \\
\cline { 2 - 3 } & Slope $\left(\mathrm{m} \mathrm{s}^{-1} \mathrm{~min}^{-1}\right)$ & $p$ value & & Slope $\left(\mathrm{m} \mathrm{s}^{-1} \mathrm{~min}^{-1}\right)$ & $p$ value \\
\hline 1 & 1.60 & 0.003 & & 1.68 & 0.000 \\
2 & 1.44 & 0.001 & 1.12 & 0.022 \\
3 & 1.68 & 0.001 & 1.28 & 0.002 \\
4 & 2.00 & 0.001 & 2.32 & 0.010 \\
5 & 1.28 & 0.004 & 2.64 & 0.000 \\
6 & 1.76 & 0.000 & & 0.80 & 0.04 \\
Mean $\pm \mathrm{SD}$ & $1.62 \pm 0.25$ & & $1.64 \pm 0.72$ & \\
\hline
\end{tabular}

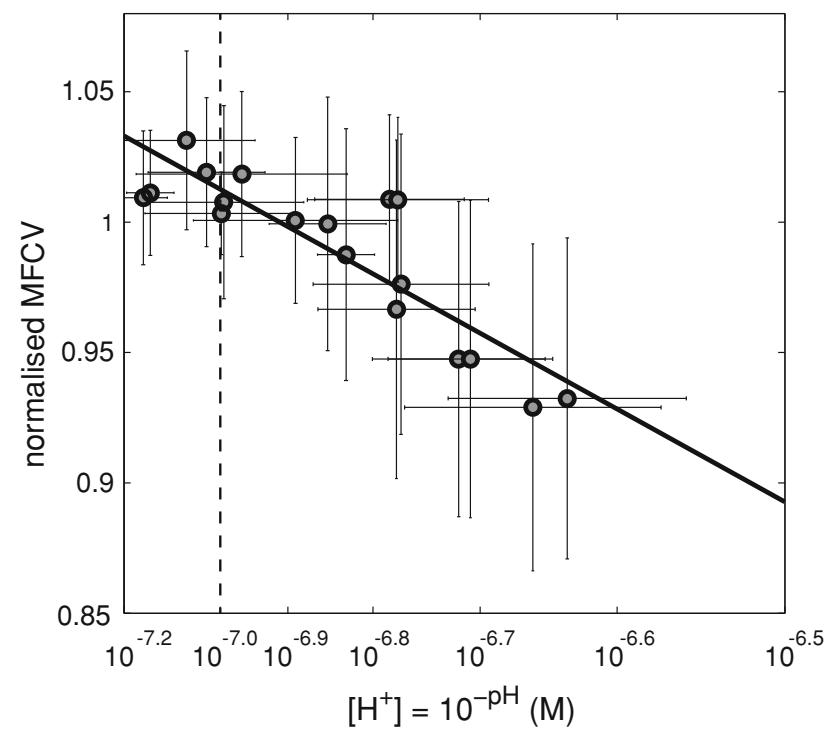

Fig. 6 Relation between normalized MFCV and muscle $\left[\mathrm{H}^{+}\right]$ $\left(\left[\mathrm{H}^{+}\right]=10^{-\mathrm{pH}}\right)$ calculated from three exercise bouts at $65-\mathrm{N}$ braking load (Fig. 5a, b solid black dots). The dashed line indicates $\mathrm{pH}=7.0$. The solid line indicates the calculated linear regression model $\left(\mathrm{MFCV}=1.068-554,227 *\left[\mathrm{H}^{+}\right]\right.$, Pearson's $\left.R=-0.87 ; p<0.001\right)$. Error bars indicate SD

the vastus medialis was activated beyond its $\mathrm{VO}_{2 \max }$. A complementary piece of information indicating the intensity of the workloads was the time the workload could be sustained. This was between $\sim 1$ min for the $65-\mathrm{N}$ workload and $\sim 4 \mathrm{~min}$ for the $35-\mathrm{N}$ workload. Task failure being developed at such a fast timescale can only be explained by intense muscle work.

Recently, it has been shown that MFCV can be estimated reliably and accurately during cycling exercise (Farina et al. 2004b). In that study, MFCV was determined by estimating the time shift between two signals in the frequency domain by minimizing the mean square error (MSE) between signals using maximum likelihood estimation (MLE). In addition, a Gaussian windowing function was applied to select only a specific part of the entire signal burst. In the present investigation a different approach has been used, i.e., the phase difference method (Houtman et al. 2003). Using this method, MFCV was estimated for individual peaks in the EMG burst and averaged afterward to yield the mean MFCV per burst. The rationale for this change was that in comparing these two methods for the two subjects, the variation induced by measurement noise for both methods was similar, whereas the phase difference method was computationally less expensive. Comparison of the variation in MFCV calculated for an individual EMG burst (see, e.g., error bars in Fig. 3) with results by others (e.g., Farina et al. 2004b) indeed indicated a similar accuracy for both methods. Moreover, it was shown that the applied method successfully detected small changes in MFCV: i.e., increasing MFCV at higher workloads (Fig. 3). Therefore, it can be concluded that, if present, a decrease in MFCV during exercise would have been detected by the applied methodology. Furthermore, data were normalized to remove variation between subjects as a result of differences in initial MFCV. Addition of the normalization step to the post-processing of the data increased the sensitivity of the methodology for detecting dynamics in MFCV.

MFCV during high-intensity bicycle exercise

MFCV has been proposed as being dependent on motor unit recruitment. An increase in MFCV at higher workloads was reported in several previous studies (Farina et al. 2004b; Macdonald et al. 2008; Sbriccoli et al. 2009). This increase has been attributed to the recruitment of larger motor units at higher exercise intensities. In good accordance with these reports, an increase in MFCV was observed with increasing workload (Fig. 3).

Although the MFCV increased at more intense workloads, during individual workloads MFCV remained constant. This observation stands not by itself, but confirms several previous reports (Macdonald et al. 2008; Masuda et al. 1999). However, other investigations reported a clear decrease in MFCV (Farina et al. 2004b; Pozzo et al. 2006; Sbriccoli et al. 2009). The decrease in MFCV almost invariably observed during moderate to high-intensity isometric contractions has been 
attributed to a combination of high-metabolic demand, fast motor unit firing rates, and diminution of the circulation with the accumulation of metabolic byproducts (Miller et al. 1995). The accumulation of extracellular potassium, especially in the T-tubulus and the accumulation of lactate together with a lowering of $\mathrm{pH}$ are believed to be the main determinants for the lowering of MFCV during isometric contractions (Zwarts and Stegeman 2003). Changes in MFCV can thus be attributed to multiple physiological factors, among them, $\mathrm{pH}$ being an important determinant. We hypothesized that the observation of the MFCV remaining constant during high-intensity cycling exercise was related to a rather mild acidosis of the muscle during this particular type of exercise (i.e., dynamic, two- legged bicycling; see also (Jeneson and Bruggeman 2004)). A first test of the proposed hypothesis was to determine the degree of acidification during the different exercise bouts. The MRS results showed a $\mathrm{pH}$ decrease during exercise of maximally 0.3 units. This drop is less than half the $\mathrm{pH}$ decreases typically observed during high-intensity isometric contractions ( $>0.6$ units) (Miller et al. 1988; Sullivan et al. 1994; Walter et al. 1999; Wilson et al. 1988) and is in good agreement with previous reports (Jeneson and Bruggeman 2004; Schmitz et al. 2010). The acidosis observed during bicycle exercise was classified as mild compared to the proton accumulation typically observed during intense isometric contractions. This observation supported part of the proposed hypothesis: i.e., a relatively small drop in $\mathrm{pH}$ during exhaustive bicycle exercise. Previously, the observation of mild acidosis during bicycle exercise was explained by a higher degree of muscle perfusion and more intense activation of the cardio respiratory system during this particular mode of exercise. Together, they cause a higher rate of lactate and $\mathrm{CO}_{2}$ removal from the muscle during bicycle exercise compared to isometric contraction, explaining the smaller drop in $\mathrm{pH}$ (Jeneson and Bruggeman 2004).

To further test the hypothesis it was investigated if an additional drop in muscle $\mathrm{pH}$ would indeed have a negative effect on MFCV. To induce such further $\mathrm{pH}$ decrease, we exploited knowledge from previous studies (Jeneson and Bruggeman 2004; Schmitz et al. 2010) that $\mathrm{pH}$ rapidly decreases an additional 0.3 units in the first $30 \mathrm{~s}$ of recovery following a high-intensity exercise bout. This additional drop in $\mathrm{pH}$ has been attributed to a decrease in cellular proton buffer capacity due to the consumption of $\mathrm{Pi}$ in oxidative phosphorylation and subsequent incorporation in the PCr buffer pool (Jeneson and Bruggeman 2004). By performing a second exercise bout after $30 \mathrm{~s}$ of recovery, we recorded MFCV during cycling exercise when cellular $\mathrm{pH}$ was about 6.5. The results of this experiment indeed confirmed a significant decrease of MFCV at this lowered $\mathrm{pH}$. It was now possible to sample the relation between MFCV and $\mathrm{pH}$ in the domain between 6.5 and 7.1 , since during the second and third exercise bout an increase in $\mathrm{pH}$ was observed. These dynamics in cellular $\mathrm{pH}$ were explained by an increasing cellular proton buffer capacity as a result of increasing Pi concentration (Jeneson and Bruggeman 2004). From the resulting complete dataset (of the 65-N exercise and following bouts) a significant correlation was calculated (Fig. 6). This result is in line with the proposed hypothesis and in good accordance with previous reports on a $\mathrm{pH}$ dependency of MFCV (Houtman et al. 2001, 2003; Linssen et al. 1996). We speculate that the inconsistency between studies reporting either an absence or presence of a decreasing MFCV during fatiguing dynamic contractions could be a result of an omnipresent relation between $\mathrm{pH}$ and MFCV, apparently most clearly present for $\mathrm{pH}$ levels below 6.8-7.0 (Figs. 5a, b, 6). A mild acidosis ( $\mathrm{pH}$ 6.8-7.0) may result in a small MFCV change even below the detection limit. Inconsistent results in literature can likely be explained by a variation in the degree of muscle acidification present between studies. This variation may arise from differences in, e.g., ergometer setup, specific exercise protocol and/or fitness level of the subjects.

\section{Conclusions}

In the present investigation, we did not detect an MFCV decrease during bicycle exercise until task failure. Combining MRS and high-density sEMG measurements, we found a significant correlation between MFCV and cellular $\mathrm{pH}$, whereby proton accumulation causes slowing down of action potential propagation along the muscle. These results confirm that the $\mathrm{MFCV}$, a non-invasive measure, can provide relevant information on physiologically significant muscle acidosis. The absence of a decrease in MFCV during bicycling exercise, observed in the present as well as in previously reported studies, can, therefore, be explained by the absence of a large drop in $\mathrm{pH}$ during this particular mode of intense upper leg exercise.

Conflict of interest The authors declare that they have no conflict of interest. The experiments conducted in this study comply with the current laws of The Netherlands.

Open Access This article is distributed under the terms of the Creative Commons Attribution Noncommercial License which permits any noncommercial use, distribution, and reproduction in any medium, provided the original author(s) and source are credited.

\section{References}

Allen DG, Lamb GD, Westerblad H (2008) Skeletal muscle fatigue: cellular mechanisms. Physiol Rev 88:287-332

Arendt-Nielsen L, Mills KR (1988) Muscle fibre conduction velocity, mean power frequency, mean EMG voltage and force during 
submaximal fatiguing contractions of human quadriceps. Eur $\mathrm{J}$ Appl Physiol Occup Physiol 58:20-25

Beck RB, O’Malley M, Van Dijk JP, Nolan P, Stegeman DF (2004) The effects of bipolar electrode montage on conduction velocity estimation from the surface electromyogram. J Electromyogr Kinesiol 14:505-514

Blok JH, Van Dijk JP, Drost G, Zwarts MJ, Stegeman DF (2002) A high-density multichannel surface electromyography system for the characterization of single motor units. Rev Sci Instrum 73:1887-1897

Farina D, Schulte E, Merletti R, Rau G, Disselhorst-Klug C (2003) Single motor unit analysis from spatially filtered surface electromyogram signals. Part I: spatial selectivity. Med Biol Eng Comput 41:330-337

Farina D, Merletti R, Enoka RM (2004a) The extraction of neural strategies from the surface EMG. J Appl Physiol 96:1486-1495

Farina D, Pozzo M, Merlo E, Bottin A, Merletti R (2004b) Assessment of average muscle fiber conduction velocity from surface EMG signals during fatiguing dynamic contractions. IEEE Trans Biomed Eng 51:1383-1393

Forbes SC, Paganini AT, Slade JM, Towse TF, Meyer RA (2009) Phosphocreatine recovery kinetics following low- and highintensity exercise in human triceps surae and rat posterior hindlimb muscles. Am J Physiol Regul Integr Comp Physiol 296:R161-R170

Fortune E, Lowery MM (2009) Effect of extracellular potassium accumulation on muscle fiber conduction velocity: a simulation study. Ann Biomed Eng 37:2105-2117

Houtman CJ, Heerschap A, Zwarts MJ, Stegeman DF (2001) pH heterogeneity in tibial anterior muscle during isometric activity studied by (31)P-NMR spectroscopy. J Appl Physiol 91:191-200

Houtman CJ, Stegeman DF, Van Dijk JP, Zwarts MJ (2003) Changes in muscle fiber conduction velocity indicate recruitment of distinct motor unit populations. J Appl Physiol 95:1045-1054

Jeneson JA, Bruggeman FJ (2004) Robust homeostatic control of quadriceps $\mathrm{pH}$ during natural locomotor activity in man. FASEB J 18:1010-1012

Jeneson JA, Schmitz JP, van den Broek NM, van Riel NA, Hilbers PA, Nicolay K, Prompers JJ (2009) Magnitude and control of mitochondrial sensitivity to ADP. Am J Physiol Endocrinol Metab 297:E774-E784

Jeneson JA, Schmitz JP, Hilbers PA, Nicolay K (2010) An MRcompatible bicycle ergometer for in-magnet whole-body human exercise testing. Magn Reson Med 63:257-261

Krogh-Lund C (1993) Myo-electric fatigue and force failure from submaximal static elbow flexion sustained to exhaustion. Eur $\mathbf{J}$ Appl Physiol Occup Physiol 67:389-401

Lapatki BG, Van Dijk JP, Jonas IE, Zwarts MJ, Stegeman DF (2004) A thin, flexible multielectrode grid for high-density surface EMG. J Appl Physiol 96:327-336

Linssen WH, Stegeman DF, Merks MJ, Binkhorst RA, Notermans SL (1996) Electromyographic evidence of delayed fatigue-induced sarcolemmal excitability impairment in McArdle's disease. J Electromyogr Kinesiol 6:147-157

Macdonald JH, Farina D, Marcora SM (2008) Response of electromyographic variables during incremental and fatiguing cycling. Med Sci Sports Exerc 40:335-344

Masuda K, Masuda T, Sadoyama T, Inaki M, Katsuta S (1999) Changes in surface EMG parameters during static and dynamic fatiguing contractions. J Electromyogr Kinesiol 9:39-46
Merletti R, Botter A, Troiano A, Merlo E, Minetto MA (2009) Technology and instrumentation for detection and conditioning of the surface electromyographic signal: state of the art. Clin Biomech (Bristol, Avon) 24:122-134

Metting van Rijn AC, Peper A, Grimbergen CA (1990) High-quality recording of bioelectric events. Part 1 . Interference reduction, theory and practice. Med Biol Eng Comput 28:389-397

Miller RG, Boska MD, Moussavi RS, Carson PJ, Weiner MW (1988) $31 \mathrm{P}$ nuclear magnetic resonance studies of high energy phosphates and $\mathrm{pH}$ in human muscle fatigue. Comparison of aerobic and anaerobic exercise. J Clin Invest 81:1190-1196

Miller RG, Kent-Braun JA, Sharma KR, Weiner MW (1995) Mechanisms of human muscle fatigue. Quantitating the contribution of metabolic factors and activation impairment. Adv Exp Med Biol 384:195-210

Pozzo M, Alkner B, Norrbrand L, Farina D, Tesch PA (2006) Musclefiber conduction velocity during concentric and eccentric actions on a flywheel exercise device. Muscle Nerve 34:169-177

Prompers JJ, Jeneson JA, Drost MR, Oomens CC, Strijkers GJ, Nicolay K (2006) Dynamic MRS and MRI of skeletal muscle function and biomechanics. NMR Biomed 19:927-953

Rodenburg JB, de Boer RW, Jeneson JA, van Echteld CJ, Bar PR (1994) 31P-MRS and simultaneous quantification of dynamic human quadriceps exercise in a whole body MR scanner. J Appl Physiol 77:1021-1029

Sbriccoli P, Sacchetti M, Felici F, Gizzi L, Lenti M, Scotto A, De Vito G (2009) Non-invasive assessment of muscle fiber conduction velocity during an incremental maximal cycling test. J Electromyogr Kinesiol 19:e380-e386

Schmitz JP, van Riel NA, Nicolay K, Hilbers PA, Jeneson JA (2010) Silencing of glycolysis in muscle: experimental observation and numerical analysis. Exp Physiol 95:380-397

Sullivan MJ, Saltin B, Negro-Vilar R, Duscha BD, Charles HC (1994) Skeletal muscle $\mathrm{pH}$ assessed by biochemical and 31P-MRS methods during exercise and recovery in men. J Appl Physiol 77:2194-2200

Taylor DJ, Styles P, Matthews PM, Arnold DA, Gadian DG, Bore P, Radda GK (1986) Energetics of human muscle: exercise-induced ATP depletion. Magn Reson Med 3:44-54

Tobin RB, Mackerer CR, Mehlman MA (1972) pH effects on oxidative phosphorylation of rat liver mitochondria. Am J Physiol 223:83-88

Vanhamme L, van den Boogaart A, Van Huffel S (1997) Improved method for accurate and efficient quantification of MRS data with use of prior knowledge. J.Magn Reson 129:35-43

Walter G, Vandenborne K, Elliott M, Leigh JS (1999) In vivo ATP synthesis rates in single human muscles during high intensity exercise. J Physiol 519((Pt 3)):901-910

Wilson JR, McCully KK, Mancini DM, Boden B, Chance B (1988) Relationship of muscular fatigue to $\mathrm{pH}$ and diprotonated $\mathrm{Pi}$ in humans: a 31P-NMR study. J Appl Physiol 64:2333-2339

Zwarts MJ, Stegeman DF (2003) Multichannel surface EMG: basic aspects and clinical utility. Muscle Nerve 28:1-17

Zwarts MJ, van Weerden TW, Haenen HT (1987) Relationship between average muscle fibre conduction velocity and EMG power spectra during isometric contraction, recovery and applied ischemia. Eur J Appl Physiol Occup Physiol 56:212-216 